\title{
Improving Coverage Estimation for Cellular Networks with Spatial Bayesian Prediction based on Measurements
}

\author{
Berna Sayrac ${ }^{\star}$, Janne Riihijärvi ${ }^{\star}$, Petri Mähönen, \\ Sana Ben Jemaa`, Eric Moulines ${ }^{\star \star}$, Sébastien Grimoud ${ }^{\star}$ \\ ${ }^{\circ}$ Orange Labs, Issy-Les-Moulineaux, France \\ *Institute for Networked Systems, RWTH Aachen University, Aachen, Germany \\ ${ }^{\star}$ Telecom ParisTech, Paris, France \\ email: berna.sayrac@orange-ftgroup.com
}

\begin{abstract}
Cellular operators routinely use sophisticated planning tools to estimate the coverage of the network based on building and terrain data combined with detailed propagation modeling. Nevertheless, coverage holes still emerge due to equipment failures, or unforeseen changes in the propagation environment. For detecting these coverage holes, drive tests are typically used. Since carrying out drive tests is expensive and time consuming, there is significant interest in both improving the quality of the coverage estimates obtained from a limited number of drive test measurements, as well as enabling the incorporation of measurements from mobile terminals. In this paper we introduce a spatial Bayesian prediction framework that can be used for both of these purposes. We show that using techniques from modern spatial statistics we can significantly increase the accuracy of coverage predictions from drive test data. Further, we carry out a detailed evaluation of our framework in urban and rural environments, using realistic coverage data obtained from an operator planning tool for an operational cellular network. Our results indicate that using spatial prediction techniques can more than double the likelihood of detecting coverage holes, while retaining a highly acceptable false alarm probability.
\end{abstract}

\section{Categories and Subject Descriptors}

C.2 [Computer-Communication Networks]: Miscellaneous; G.3 [Probability and Statistics]: Statistical computing; I.6.4 [Simulation and Modeling]: Model Validation and Analysis; I.6.5 [Simulation and Modeling]: Model Development

\section{Keywords}

Coverage estimation, minimization of drive tests, Bayesian kriging, spatial statistics

Permission to make digital or hard copies of all or part of this work for personal or classroom use is granted without fee provided that copies are not made or distributed for profit or commercial advantage and that copies bear this notice and the full citation on the first page. To copy otherwise, to republish, to post on servers or to redistribute to lists, requires prior specific permission and/or a fee.

CellNet'12, August 13, 2012, Helsinki, Finland.

Copyright 2012 ACM 978-1-4503-1475-6/12/08 ...\$15.00.

\section{INTRODUCTION}

Coverage estimation is one of the key problems in deployment and operation of cellular networks. While sophisticated planning tools incorporating building and terrain aware propagation models typically result in accurate coverage estimates, coverage holes are nevertheless occasionally formed. These can result from changes in the propagation environment, equipment failures, or other causes. Drive tests are then used to discover these coverage holes, either as routine part of network diagnostics, or in a targeted fashion based on information obtained from the customers and statistics gathered from the network. However, drive tests are in general both expensive and time consuming. Therefore, operators have keen interest in both maximizing the utility of information obtained from drive tests, and also minimizing the need for them. To achieve the latter, recent work in 3GPP has targeted harnessing the measurement capabilities of mobile terminals for the purposes of network diagnostics. The related work item goes by the name of "minimization of drive tests" (MDT) [1].

In this paper we show how coverage estimation based on either drive tests or data obtained through the MDT approach can be improved using Bayesian spatial statistics [7]. This extends our earlier work on spatial estimation problems in wireless networks $[2,10]$, and also illustrates how radio environment map (REM) techniques $[9,12]$ can be applied in the cellular network context as discussed in [3]. While conventional drive tests can only uncover coverage holes in regions where the measurements are actively conducted, our approach can estimate their presence in adjacent regions as well, based on the use of spatial prediction techniques. The Bayesian nature of our scheme also allows to take into account prior knowledge the operator has on their network setup, increasing the accuracy of the results. We show using realistic coverage data that spatial prediction techniques can significantly increase the likelihood of discovering coverage holes for a given number of measurements conducted, while retaining low probability of false alarms.

The rest of this paper is structured as follows. In Section 2 we give an overview of our spatial estimation approach, including the necessary mathematical foundations. We then introduce in Section 3 our evaluation scenarios, including the coverage data sets used together with the metrics applied to quantify the performance of our scheme. Results of the performance evaluation are given in Section 4, and conclusions in Section 5. 


\section{COVERAGE PREDICTION WITH BAYESIAN KRIGING}

We consider the DownLink (DL) transmission of a cellular radio access where there is only one BaseStation (BS) transmitter equipped with an omnidirectional antenna. Let $y\left(\mathbf{x}_{i}\right)$ denote the DL received power (in $\mathrm{dB}$ ) at location $\mathbf{x}_{i}$. Assuming that the fast fading effects are averaged out by the receivers, $y\left(\mathbf{x}_{i}\right)$ can be expressed as:

$$
y\left(\mathbf{x}_{i}\right)=p_{0}-10 \alpha \log _{10} d_{i}+s\left(\mathbf{x}_{i}\right)+z_{i},
$$

where $p_{0}$ is the transmitted power (in $\mathrm{dBm}$ ), $\alpha$ is the pathloss coefficient, $d_{i}$ is the distance between the transmitter and the receiver location $\mathbf{x}_{i}, s\left(\mathbf{x}_{i}\right)$ is the shadowing (in $\mathrm{dB}$ ), and $z_{i}$ is the zero-mean noise term which incorporates the uncertainties of the measurement process and all other random effects due to the propagation environment.

Equation (1) is the well-known channel model in the $\mathrm{dB}$ scale, which models the wireless channel as the sum of a deterministic linear pathloss term and two stochastic terms: shadowing and noise. This model is one of the most widely used wireless channel models due to its simplicity and to its overall ability to represent the main characteristics of the wireless channel behaviour in a variety of important wireless environments. The random noise process is assumed to consist of independent and identically distributed Gaussian samples, which are also independent of the shadowing term. Shadowing is a zero-mean Gaussian random variable that is spatially correlated according to the exponential correlation model [5]

$$
\mathbb{E}\left\{s\left(\mathbf{x}_{i}\right), s\left(\mathbf{x}_{j}\right)\right\}=r_{i j}=\frac{1}{\theta} \exp \left(-\frac{d_{i j}}{\phi}\right),
$$

where $\frac{1}{\theta}$ is the shadowing variance in $\mathrm{dB}, d_{i j}$ is the distance between locations $\mathbf{x}_{i}$ and $\mathbf{x}_{j}$ and $\phi$ is the correlation distance of the shadowing.

Such power measurements are carried out by a set of $N$ receiving terminals, located at the set of locations $\mathbf{x}=$ $\left\{\mathbf{x}_{1}, \mathbf{x}_{2}, \ldots, \mathbf{x}_{N}\right\}$. Arranging these measurements in a $N \times 1$ column vector $\mathbf{y}(\mathbf{x})$, we obtain the vector-matrix relation

$$
\mathbf{y}=X \beta+\mathbf{u}
$$

where

$$
X=\left[\begin{array}{cc}
1 & -10 \log _{10}\left(d_{1}\right) \\
\vdots & \vdots \\
1 & -10 \log _{10}\left(d_{N}\right)
\end{array}\right], \beta=\left[\begin{array}{c}
p_{0} \\
\alpha
\end{array}\right], \mathbf{u}=\left[\begin{array}{c}
s\left(\mathbf{x}_{1}\right)+z_{1} \\
\vdots \\
s\left(\mathbf{x}_{N}\right)+z_{N}
\end{array}\right]
$$

Here, $X$ is a $N \times 2$ deterministic matrix of known functions of the measurement locations $\mathbf{x}, \beta$ is the $2 \times 1$ parameter vector of the spatial mean and $\mathbf{u}$ is a $N \times 1$ multivariate Gaussian vector whose covariance matrix is $Q_{y y}(\theta, \phi, \tau)=\frac{1}{\theta}\left(R_{y y}(\phi)+\right.$ $\left.\tau I_{N}\right)$ where $\frac{1}{\theta} R_{y y}(\phi)$ is the $N \times N$ covariance matrix of the spatially correlated shadowing term whose $(i, j)^{\text {th }}$ entry is equal to $r_{i j}$ of equation (2), $I_{N}$ is the $N \times N$ identity matrix and $[.]^{T}$ denotes vector/matrix transpose. Note that the variance of the noise process is $\frac{\tau}{\theta}$. Note also that $\mathbf{y}, X$ and $\mathbf{u}$ are functions of the locations $\mathbf{x}$. The aim is to predict the received power values at locations where we do not have measurements. Let $\mathrm{x}_{0}$ denote the $M \times 1$ vector of those locations. The same underlying model is assumed for the predictions, given by

$$
\mathbf{y}_{0}=X_{0} \beta+\mathbf{u}_{0},
$$

where $\mathbf{y}_{0}$ is the $M \times 1$ vector of received power values at locations $\mathbf{x}_{0}, X_{0}$ is the $M \times 2$ matrix of deterministic effects for $\mathbf{y}_{0}$ and $\mathbf{u}_{0}$ is the $M \times 1$ stochastic vector whose covariance matrix is denoted by $Q_{00}(\theta, \phi, \tau)$. Note that for notational convenience, the dependence of $\mathbf{y}, X$ and $\mathbf{u}\left(\mathbf{y}_{0}, X_{0}\right.$ and $\mathbf{u}_{0}$ resp.) on $\mathbf{x}$ ( $\mathbf{x}_{0}$ resp.) is not shown in equations (3) and (5).

The model parameters $\beta, \theta, \phi$ and $\tau$ are unknown. Therefore, they have to be estimated from the existing measurement dataset. However, those parameters are not completely unknown to us: we have some prior knowledge on their probable values. For example, we can say that the radiated power $p_{0}$ is close to the power at the antenna feeder, the propagation pathloss coefficient $\alpha$ is around 3.5 in urban areas [6] and the shadowing standard deviation $1 / \sqrt{\theta}$ typically ranges between 8 and $11 d B$ for typical outdoor Above RoofTop to Below RoofTop scenarios [11]. Including those prior information into the model helps us enhance the prediction quality, so we prefer using a Bayesian approach in this paper. Besides, the Gaussian assumption of the stochastic component vector $\mathbf{u}$ in the chosen model provides ease and tractability in the complex analytical derivations of the Bayesian inference framework.

The task of predicting the received power values at locations $\mathbf{x}_{0}$ is equivalent to finding an estimator $\hat{\mathbf{y}}_{0}$ of the random vector $\mathbf{y}_{0}$ given measurements $\mathbf{y}$. This estimator is preferably a linear function of measurements $\mathbf{y}$ which minimizes a given loss/risk/cost function. In Bayesian context, this is equivalent to the Bayes estimator which minimizes a given posterior expected loss/risk/cost (a.k.a. Bayes risk). The most commonly used risk function is the squared error risk (or the Mean Squared Error - MSE) for which the Bayes estimator is the posterior mean $\mathbb{E}\left\{\mathbf{y}_{0} \mid \mathbf{y}\right\}$ given by

$$
\hat{\mathbf{y}}_{0}=\arg \min _{\mathbf{y}_{0}^{*}} \mathbb{E}\left\{\left(\mathbf{y}_{0}-\mathbf{y}_{0}^{*}\right)^{T}\left(\mathbf{y}_{0}-\mathbf{y}_{0}^{*}\right) \mid \mathbf{y}\right\},=\mathbb{E}\left\{\mathbf{y}_{0} \mid \mathbf{y}\right\},
$$

and the Bayes risk (or MSE) is the posterior variance:

$$
\frac{1}{M} \mathbb{E}\left\{\left(\mathbf{y}_{0}-\hat{\mathbf{y}}_{0}\right)^{T}\left(\mathbf{y}_{0}-\hat{\mathbf{y}}_{0}\right) \mid \mathbf{y}\right\}=\frac{1}{M} \operatorname{var}\left\{\mathbf{y}_{0} \mid \mathbf{y}\right\}
$$

For the MSE Bayes estimator, we need to calculate the marginal posterior (or Bayesian) pdf $p\left(\mathbf{y}_{0} \mid \mathbf{y}\right)$, or at least its moments such as the posterior mean $\mathbb{E}\left\{\mathbf{y}_{0} \mid \mathbf{y}\right\}$ and covariance $\operatorname{cov}\left\{\mathbf{y}_{0} \mid \mathbf{y}\right\}$. The derivation of these quantities are quite lengthy, therefore we will only give the main points in the following and omit the details due to space limitations.

We start by writing the Bayesian pdf $p\left(\mathbf{y}_{0} \mid \mathbf{y}\right)$ as

$$
\begin{aligned}
p\left(\mathbf{y}_{0} \mid \mathbf{y}\right)=\iiint_{\tau, \phi, \theta, \beta} \int_{0} p\left(\mathbf{y}_{0} \mid \beta, \theta, \phi, \tau, \mathbf{y}\right) & \\
& \times p(\beta, \theta, \phi, \tau \mid \mathbf{y}) d \beta d \theta d \phi d \tau .
\end{aligned}
$$

The first integrand in equation (8) is the conditional pdf of $\mathbf{y}_{0}$ given $\mathbf{y}$ and the model parameters. Assuming that $Q_{y y}$ is non-singular, it can be shown that this pdf is Gaussian with mean $X_{0} \beta+Q_{0 y} Q_{y y}^{-1}(\mathbf{y}-X \beta)$ and covariance matrix $Q_{00}-Q_{0 y} Q_{y y}^{-1} Q_{y 0}$ [4] where $Q_{0 y}$ and $Q_{y 0}$ are the crosscovariance matrices between $\mathbf{y}_{0}$ and $\mathbf{y}$. Note that for notational convenience, the dependence of $Q_{0 y}$ and $Q_{y 0}$ on the model parameters is not shown. 
The second integrand in equation (8) is the joint posterior of the model parameters which can be decomposed into the product of two joint posteriors:

$$
p(\beta, \theta, \phi, \tau \mid \mathbf{y})=p(\beta, \theta \mid \phi, \tau, \mathbf{y}) p(\phi, \tau \mid \mathbf{y}) .
$$

The first joint posterior $p(\beta, \theta \mid \phi, \tau, \mathbf{y})$ is proportional to the product of a likelihood term and a prior as in

$$
p(\beta, \theta \mid \phi, \tau, \mathbf{y}) \propto p(\mathbf{y} \mid \beta, \theta, \phi, \tau) p(\beta, \theta \mid \phi, \tau),
$$

where $p(\beta, \theta \mid \phi, \tau)$ is the joint (conditional) prior of $\beta$ and $\theta$, and $p(\mathbf{y} \mid \beta, \theta, \phi, \tau)$ is the likelihood term which is Gaussian with mean $\mathbf{y}-X \beta$ and covariance $Q_{y y}$.

An important issue in Bayesian inference is the choice of prior distributions. It is common practice to choose functional forms that facilitate the involved analytical treatments. Such functional forms are called as conjugate priors, meaning conjugate to the likelihood function, such that the posterior distribution has the same functional form as the prior distribution. Conjugate priors have been identified for the most widely used distribution functions [8].

Reformulating the likelihood term $p(\mathbf{y} \mid \beta, \theta, \phi, \tau)$ and matching the pdf parameters through several algebraic manipulations (whose details are omitted here for brevity reasons), the conjugate prior for $p(\beta, \theta \mid \phi, \tau)$ can be found as the Normal-Gamma-2 density [8]. The Normal-Gamma-2 density represents a multivariate Gaussian random vector $\beta$ whose covariance matrix is scaled with a random variable whose inverse $(\theta)$ is Gamma-2 distributed. This implies that $p(\beta \mid \theta, \phi, \tau)$ is Gaussian and $p(\theta \mid \phi, \tau)$ is Gamma-2 distributed.

The choice of the conjugate prior yields the same functional form for the joint posterior, so $p(\beta, \theta \mid \phi, \tau, \mathbf{y})$ is also Normal-Gamma-2. Its exact expression (and moments) can be calculated using equation (10) which is followed by several algebraic manipulations that are again skipped here for brevity purposes.

As for the second joint posterior of equation (9), $p(\phi, \tau \mid \mathbf{y})$, it can be calculated by combining the Gaussian likelihood $p(\mathbf{y} \mid \beta, \theta, \phi, \tau)$ and the two Normal-Gamma-2 pdfs, $p(\beta, \theta \mid \phi, \tau)$, $p(\beta, \theta \mid \phi, \tau, \mathbf{y})$ with the following expression:

$$
p(\phi, \tau \mid \mathbf{y})=\frac{p(\mathbf{y} \mid \beta, \theta, \phi, \tau) p(\beta, \theta \mid \phi, \tau)}{p(\beta, \theta \mid \phi, \tau, \mathbf{y})} p(\phi, \tau) .
$$

The joint prior $p(\phi, \tau)$ can be written as the product of the marginal densities $p(\phi)$ and $p(\tau)$, since the noise is assumed independent of the shadowing. Furthermore, we assume discrete pdfs for $p(\phi)$ and $p(\tau)$. Therefore,

$$
p(\phi, \tau)=\sum_{k} \sum_{l} \phi_{k} \tau_{l} \delta\left(\phi-\phi_{k}\right) \delta\left(\tau-\tau_{l}\right),
$$

where $\delta($.$) is the Dirac delta function. Thus, replacing equa-$ tion (12) in equation (11), $p(\phi, \tau \mid \mathbf{y})$ can also be calculated as a discrete pdf, i.e. a weighted sum of delta functions.

We can now go back to the Bayesian pdf of equation (8). Applying the chain rule to $p(\beta, \theta, \phi, \tau \mid \mathbf{y})$, it can be written as

$$
\begin{gathered}
p\left(\mathbf{y}_{0} \mid \mathbf{y}\right)=\iint_{\tau, \phi, \theta}\left[\int_{\beta} p\left(\mathbf{y}_{0} \mid \beta, \theta, \phi, \tau, \mathbf{y}\right) p(\beta \mid \theta, \phi, \tau, \mathbf{y}) d \beta\right] \\
\times p(\theta \mid \phi, \tau, \mathbf{y}) p(\phi, \tau \mid \mathbf{y}) d \theta d \phi d \tau .
\end{gathered}
$$

The inner integral, $p\left(\mathbf{y}_{0} \mid \theta, \phi, \tau, \mathbf{y}\right)$, can be recognized as the marginalisation (wrt $\beta$ ) of a joint Gaussian pdf. Therefore, it is also Gaussian whose mean and variance can be easily calculated by analytical integration. Proceeding with the integration with respect to $\theta$, we can write

$$
\begin{aligned}
& p\left(\mathbf{y}_{0} \mid \mathbf{y}\right)= \\
& \iint_{\tau, \phi}\left[\int_{\theta} p\left(\mathbf{y}_{0} \mid \theta, \phi, \tau, \mathbf{y}\right) p(\theta \mid \phi, \tau, \mathbf{y}) d \theta\right] p(\phi, \tau \mid \mathbf{y}) d \phi d \tau .
\end{aligned}
$$

Here, the inner integral can be recognized as a Student distribution whose mean $\mathbb{E}\left\{\mathbf{y}_{0} \mid \phi, \tau, \mathbf{y}\right\}$ and covariance matrix $\operatorname{cov}\left\{\mathbf{y}_{0} \mid \phi, \tau, \mathbf{y}\right\}$ can be calculated analytically [8]. These statistics can be readily used in equation (14) together with the discrete posterior $p(\phi, \tau \mid \mathbf{y})$ to calculate the posterior mean and covariance of the MSE Bayes estimator given by

$$
\mathbb{E}\left\{\mathbf{y}_{0} \mid \mathbf{y}\right\}=\sum_{\phi, \tau} p(\phi, \tau \mid \mathbf{y}) \mathbb{E}\left\{\mathbf{y}_{0} \mid \phi, \tau, \mathbf{y}\right\}
$$

and

$$
\begin{array}{r}
\operatorname{cov}\left\{\mathbf{y}_{0} \mid \mathbf{y}\right\}=\sum_{\phi, \tau} p(\phi, \tau \mid \mathbf{y})\left[\operatorname{cov}\left\{\mathbf{y}_{0} \mid \phi, \tau, \mathbf{y}\right\}+\left(\mathbb{E}\left\{\mathbf{y}_{0} \mid \phi, \tau, \mathbf{y}\right\}\right.\right. \\
\left.\left.-\mathbb{E}\left\{\mathbf{y}_{0} \mid y\right\}\right)\left(\mathbb{E}\left\{\mathbf{y}_{0} \mid \phi, \tau, \mathbf{y}\right\}-\mathbb{E}\left\{\mathbf{y}_{0} \mid \mathbf{y}\right\}\right)^{T}\right]
\end{array}
$$

Estimation of the model parameters $\beta$ and $\theta$ is carried out by calculating their posterior expectations, obtained from

$$
\mathbb{E}\{\beta \mid \mathbf{y}\}=\iint_{\phi, \tau} \mathbb{E}\{\beta \mid \phi, \tau, \mathbf{y}\} p(\phi, \tau \mid \mathbf{y}) d \phi d \tau
$$

and

$$
\mathbb{E}\{\theta \mid \mathbf{y}\}=\iint_{\phi, \tau} \mathbb{E}\{\theta \mid \phi, \tau, \mathbf{y}\} p(\phi, \tau \mid \mathbf{y}) d \phi d \tau .
$$

The marginal posteriors $p(\beta \mid \phi, \tau, \mathbf{y})$ and $p(\theta \mid \phi, \tau, \mathbf{y})$ needed to calculate $\mathbb{E}\{\beta \mid \phi, \tau, \mathbf{y}\}$ and $\mathbb{E}\{\theta \mid \phi, \tau, \mathbf{y}\}$ can be obtained by integrating $p(\beta, \theta \mid \phi, \tau, \mathbf{y})$ with respect to $\theta$ and $\beta$ respectively. The former integration results in a multivariate Student distribution while the latter yields a Gamma-2 distribution whose first two moments can be analytically calculated [8].

Considering the discrete nature of $p(\phi, \tau \mid \mathbf{y})$, the two integrals of equations (17) and (18) become weighted sums of pdfs, hence the posterior parameter expectations become weighted averages of the conditional parameter expectations given by

$$
\mathbb{E}\{\beta \mid \mathbf{y}\}=\sum_{\phi_{k}} \sum_{\tau_{l}} p\left(\phi_{k}, \tau_{l} \mid \mathbf{y}\right) \mathbb{E}\left\{\beta \mid \phi_{k}, \tau_{l}, \mathbf{y}\right\},
$$

and

$$
\mathbb{E}\{\theta \mid \mathbf{y}\}=\sum_{\phi_{k}} \sum_{\tau_{l}} p\left(\phi_{k}, \tau_{l} \mid \mathbf{y}\right) \frac{1}{\mathbb{E}\left\{\theta \mid \phi_{k}, \tau_{l}, \mathbf{y}\right\}} .
$$

\section{EVALUATION SCENARIO AND METRICS}

We shall now introduce the evaluation scenarios and metric used to evaluate the performance of the proposed spatial prediction scheme for coverage estimation. As a foundation for our evaluation work, we have used signal strength maps obtained from a highly accurate planning tool used also for operational network planning within Orange. The coverage maps are for an operational $3 \mathrm{G}$ network and the received 

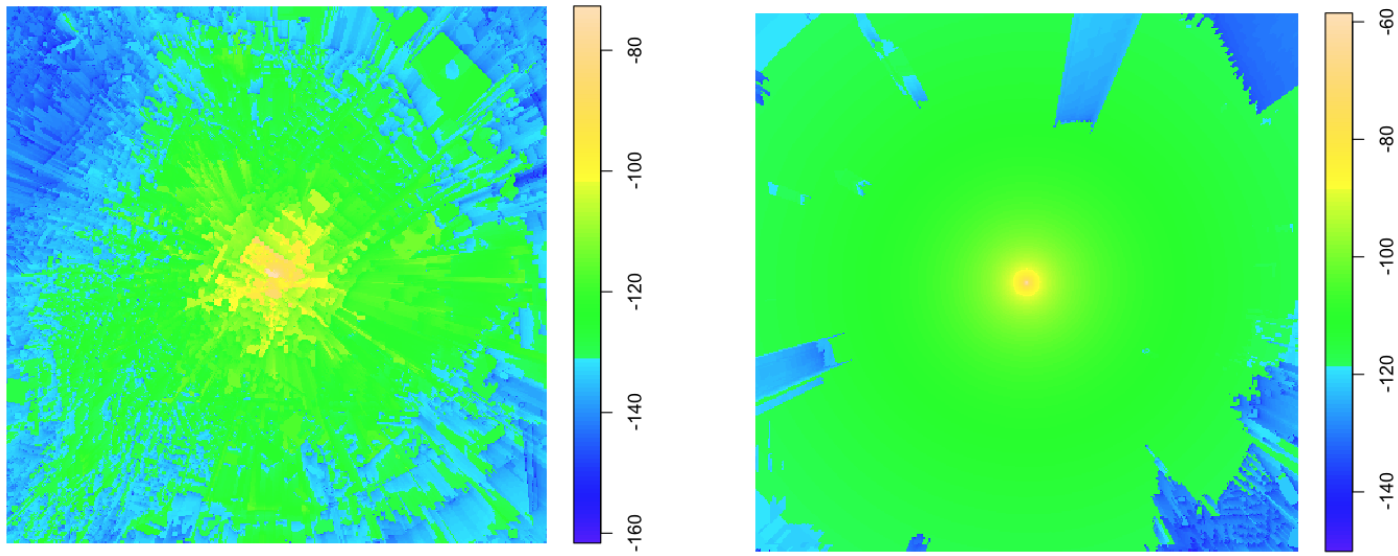

Figure 1: An illustration of the received signal strengths measured in $\mathrm{dBm}$ for the urban (left) and rural (right) evaluation scenarios. Both figures correspond to a square of $2 \mathrm{~km} \times 2 \mathrm{~km}$ in size.

\section{predictions/measurements $=1$}

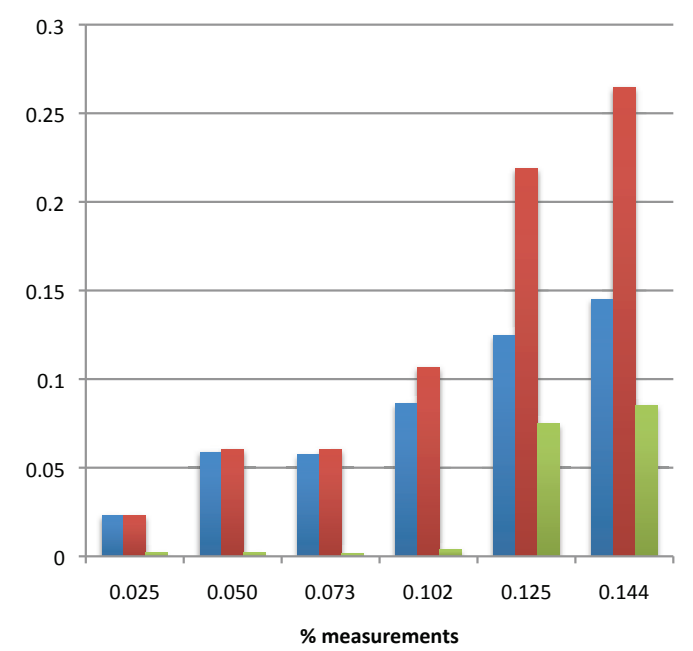

predictions/measurements $=3$

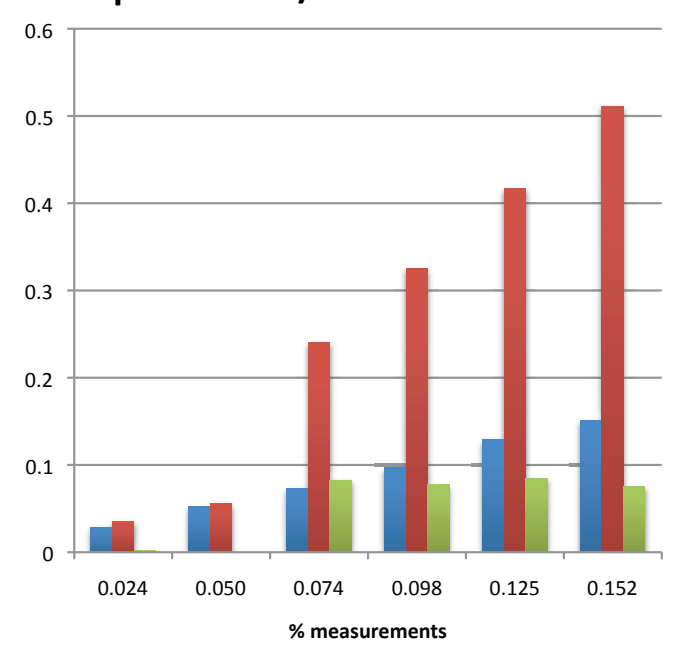

Figure 2: Detection probabilities for coverage holes using measurements only (left columns) and using both measurements and results from the spatial prediction approach (middle columns) for the urban scenario with $d_{\max }=400 \mathrm{~m}$ for $k=1$ and $k=3$. Right columns give the corresponding false alarm probabilities.

signal strength is the Received Signal Code Power (RSCP). These maps have been computed taking into account terrain shapes and buildings, and the obtained results have been repeatedly validated through drive tests. Therefore we expect them to match very closely the actual cellular network coverage. We consider in this paper two scenarios illustrated in Figure 1. First of these corresponds to an urban region just south of downtown Paris, France, while the second one corresponds to a more rural setting some $20 \mathrm{~km}$ away from Paris.

For both of these scenarios, we carry out a performance evaluation as follows. We assume that $N_{\text {meas }}$ measurements are carried out randomly at distances ranging from $d_{\text {min }}$ to $d_{\max }$ from the base station located at the centre of the region. Based on these measurements, we make predictions at $N_{\text {pred }}$ locations, chosen not to coincide with the measurement locations, but otherwise taken randomly from the same region. We then compare the measured and predicted values for the received signal strength against a reference threshold of $-118 \mathrm{dBm}$ to determine whether these points are considered to be a part of a coverage hole or not. From these results we then form the empirical probabilities for coverage hole detection (corresponding to the case for which the predictions are correctly determined to be in outage), as well as false alarms (predictions being incorrectly determined as being in outage).

In order to obtain as comprehensive evaluation results for our scheme as possible, we have covered a large number of different combinations of the parameters introduced above. For the urban scenario, two regions were considered, corre- 

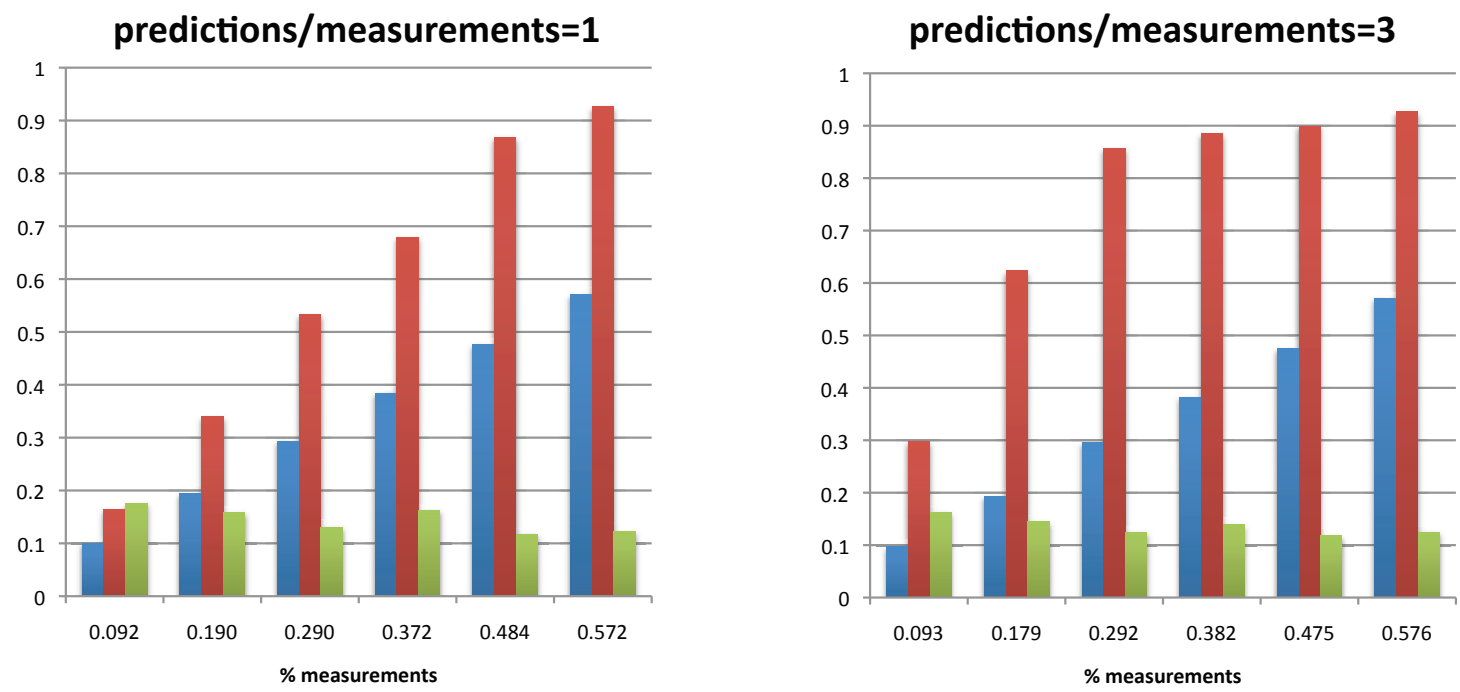

Figure 3: Detection probabilities for coverage holes using measurements only (left columns) and using both measurements and results from the spatial prediction approach (middle columns) for the urban scenario with $d_{\min }=265 \mathrm{~m}$ and $d_{\max }=335 \mathrm{~m}$, for both $k=1$ and $k=3$. Right columns give the corresponding false alarm probabilities.
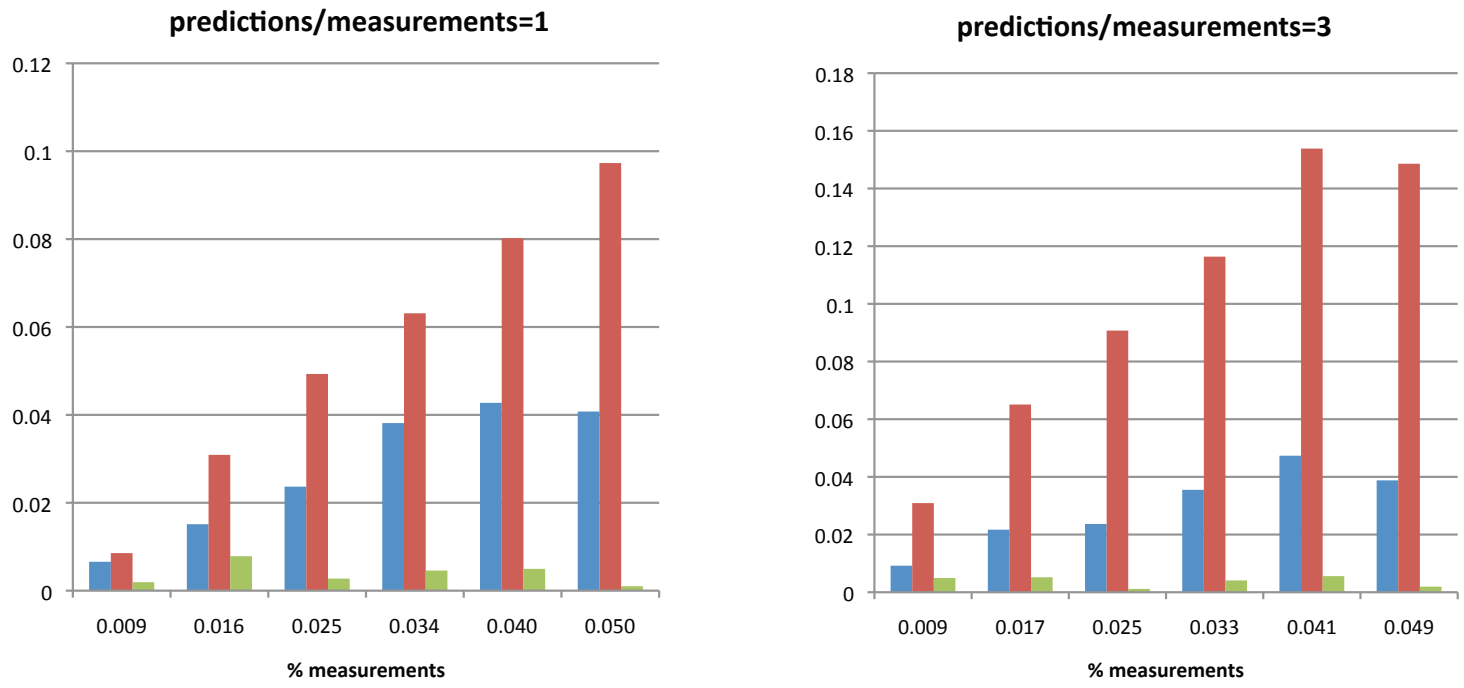

Figure 4: Detection probabilities for coverage holes using measurements only (left columns) and using both measurements and results from the spatial prediction approach (middle columns) for the rural scenario with $d_{\min }=400 \mathbf{m}$ and $d_{\max }=800 \mathbf{m}$, for both $k=1$ and $k=3$. Right columns give the corresponding false alarm probabilities.

sponding to the disk $d_{\min }=0 \mathrm{~m}$ and $d_{\max }=400 \mathrm{~m}$, and the annulus $d_{\min }=265 \mathrm{~m}$ and $d_{\max }=335 \mathrm{~m}$. For the rural case $d_{\min }$ was set to $400 \mathrm{~m}$, and $d_{\max }$ was varied from $800 \mathrm{~m}$ to $1600 \mathrm{~m}$. The numbers of measurement and prediction locations were chosen so that $N_{\text {pred }}=k \times N_{\text {meas }}$, with $k \in\{1,2,3\}$ and $N_{\text {meas }} \in\{500,1000, \ldots, 3000\}$.

\section{RESULTS}

We begin by studying the results for the urban scenario. Figure 2 shows the results for the case with $d_{\max }=400 \mathrm{~m}$ for two different ratios of the number of measurement and prediction points. We see that if the number of prediction and measurement points is equal, for small number of measurement points the gains spatial prediction yields are very small. However, as the number of measurement points is 
increased, the gains from spatial prediction increase rapidly. However, the overall detection probability remains low. As the number of prediction points is increased (corresponding to the right panel of Figure 2) the spatial prediction approach starts to perform very well, over tripling the detection probability for coverage holes compared to the measurements only-approach. We also see that the false alarm probability remains stable.

Figure 3 shows the results for the urban scenario where measurements and predictions are carried out in the annulus with $d_{\min }=265 \mathrm{~m}$ and $d_{\max }=335 \mathrm{~m}$. This corresponds to drive tests carried out specifically at the cell edge, where coverage holes are most likely to occur. We see that especially for larger ratio of number of prediction points to measurement points the spatial prediction scheme yields approximately $90 \%$ detection probability for coverage holes, with significantly improved performance compared to measurements only approach.

Figure 4 shows the results for the rural scenario focusing on the annulus with $d_{\min }=400 \mathrm{~m}$ and $d_{\max }=800 \mathrm{~m}$. The overall detection probabilities are lower than for the urban case (due to the same number of measurement points being used to cover much larger area compared to the urban case), but the gains from the spatial prediction approach are still very clear.

\section{CONCLUSIONS}

In this paper we studied the use of a spatial Bayesian prediction framework for improving coverage estimates for cellular networks based on measurements. Our results indicate that the use of techniques from modern spatial statistics can significantly increase the accuracy of coverage predictions from drive test data. We are currently extending our evaluation work with additional scenarios, including measurements from live cellular systems.

\section{Acknowledgments}

We thank EU for providing partial funding of this work through the FARAMIR project. JR and PM also thank RWTH Aachen University and DFG for partial financial support through the UMIC research centre.

\section{REFERENCES}

[1] 3GPP. TS 37.320 v10.0.0, "Radio measurement collection for Minimization of Drive Tests (MDT);Overall description; Stage 2".
[2] A. Alaya-Feki, S. Ben Jemaa, B. Sayrac, P. Houze, and E. Moulines. Informed spectrum usage in cognitive radio networks: Interference cartography. In Proceedings of IEEE PIMRC 2008, pages 1-5. IEEE, 2008.

[3] T. Cai, J. van de Beek, B. Sayrac, S. Grimoud, J. Nasreddine, J. Riihijärvi, and P. Mähönen. Design of layered radio environment maps for ran optimization in heterogeneous LTE systems. In Proceedings of IEEE PIMRC 2011, pages 172-176. IEEE, 2011.

[4] M. L. Eaton. Multivariate Statistics: a Vector Space Approach. John Wiley and Sons, 1983.

[5] M. Gudmundson. Correlation model for shadow fading in mobile radio systems. Electronics Letters, 27:2145-2146, 1991.

[6] H.-S. Jo and J.-G. Yook. Path loss characteristics for IMT-advanced systems in residential and street environments. IEEE Antennas and Wireless Propagation Letters, pages 867-871, 2010.

[7] H. Omre. Bayesian kriging - merging observations and qualified guesses in kriging. Mathematical Geology, 19(1):25-39, 1987.

[8] H. Raiffa and R. Schlaifer. Applied Statistical Decision Theory. Wiley, 2000.

[9] J. Riihijärvi, P. Mähönen, M. Petrova, and V. Kolar. Enhancing cognitive radios with spatial statistics: From radio environment maps to topology engine. In Proceedings of CROWNCOM'09, pages 1-6, 2009.

[10] J. Riihijärvi, P. Mähönen, M. Wellens, and M. Gordziel. Characterization and modelling of spectrum for dynamic spectrum access with spatial statistics and random fields. In Proceedings of IEEE PIMRC 2008, pages 1-6. IEEE, 2008.

[11] G. Senarath, W. Tong, P. Zhu, H. Zhang, D. Steer, D. Yu, M. Naden, and D. Kitchener. Multi-hop relay system evaluation methodology (channel model and performance metric). IEEE C802. 16j-06/013r3, 2007.

[12] Y. Zhao, L. Morales, J. Gaeddert, K. Bae, J. Um, and J. Reed. Applying radio environment maps to cognitive wireless regional area networks. In Proceedings of IEEE DySPAN 200\%, pages 115-118. IEEE, 2007. 